\title{
Mass Transfer from a Vertical Flat Plate due to a Constant Upward Flow
}

\author{
David McDonnell \\ Technological University Dublin, david.mcdonnell@tudublin.ie \\ Brendan Redmond \\ Technological University Dublin, brendan.redmond@tudublin.ie \\ Lawrence J. Crane \\ Institute for Numerical and Computational Analysis, Ic@incaireland.org
}

Follow this and additional works at: https://arrow.tudublin.ie/scschmatart

Part of the Mathematics Commons

\section{Recommended Citation}

D. McDonnell, B. Redmond and L.J. Crane, (2013) Mass Transfer from a Vertical Flat Plate due to a Constant Upward Flow, Zeitschrift fuer Angewandte Mathematik und Mechanik (ZAMM), 95, No. 1, 111-116, 2013. DOI: 10.1002/zamm.201100163

This Article is brought to you for free and open access by the School of Mathematics at ARROW@TU Dublin. It has been accepted for inclusion in Articles by an authorized administrator of ARROW@TU Dublin. For more information, please contact arrow.admin@tudublin.ie, aisling.coyne@tudublin.ie,gerard.connolly@tudublin.ie.

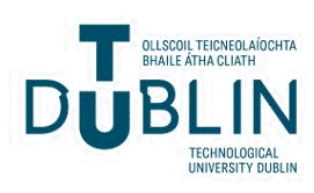




\title{
Mass Transfer from a Vertical Flat Plate due to a Constant Upward Flow
}

\author{
D. McDonnell ${ }^{* 1}$, B. Redmond ${ }^{1}$, and L.J. Crane ${ }^{2}$ \\ ${ }^{1}$ School of Mathematical Sciences, Dublin Institute of Technology, Kevin Street, Dublin 8, Ireland. \\ 2 Institute for Numerical and Computational Analysis, 7-9 Dame Court, Dublin 2, Ireland.
}

Received 15 November 2011, revised 30 November 2003, accepted 2 December 2003

Published online 3 December 2003

Key words Mass Transfer, Vertical Plate, Forced Convection, Boundary Layer Separation

Subject classification 76R50 76R05 76M45 76D10

This paper examines the mass transfer from the vertical flat surface of a soluble material due to a constant upward flow. The mass transfer rate due to this upward flow is calculated and used to obtain the distance along the surface at which the boundary layer separates. For relatively large velocities no separation will occur and the solution approaches that of forced convection on a horizontal surface.

\section{Introduction}

The aim of this paper is to examine the concentration boundary layer formed on a vertical flat surface due to a constant upward flow. The surface is composed of a soluble material placed in a liquid medium. The emphasis is on estimating the point of separation of the boundary layer. Mass transfer will occur due to the upward flow, leading to an increase in density of the dissolution medium close to the surface. At some level along the surface the weight of these dissolved particles will counteract the upward force causing boundary layer separation. For the case of sufficiently large upward velocities separation will not occur over the length of the plate, and as the upward velocity approaches infinity the effect of the dissolved particles will be negligent. In the case of lower velocities, separation will occur at some distance along the plate.

In a liquid, molecules diffuse much more slowly than momentum. Consequently, the concentration boundary layer is an order of magnitude thinner then the momentum layer. The concentration boundary layer therefore occupies the region of the momentum boundary layer close to the surface in which the velocity gradient is linear. This model is therefore analogous to that of heat transfer for large Prandtl numbers, for which an exact solution for horizontal flat plate flow exists due to Lévêque [1]. A correction term which represents the shear stress due to the mass of particles dissolved is introduced to give an expression for the net shear stress. This expression is inserted into the Lévêque solution and solved to give the overall shear stress as a function of the upward distance from the lower edge of the plate. The total mass transfer due to the upward flow is then calculated and compared with that of a corresponding horizontal flow.

\section{Point of Separation of Boundary Layer}

The concentration boundary layer equation is

$$
u \frac{\partial c}{\partial x}+v \frac{\partial c}{\partial y}=D \frac{\partial^{2} c}{\partial y^{2}}
$$

where $x$ is the height above the leading edge, $y$ is the distance from the wall, $u$ and $v$ are the components of velocity in the $x$ and $y$ directions respectively, $c$ is the concentration of dissolved particles and $D$ is the coefficient of diffusion of the soluble material. The concentration layer thickness is $\delta_{c}$ and within this thin layer the velocity gradient is taken to be $\beta$, where

$$
\beta=\left(\frac{\partial u}{\partial y}\right)_{y=0}
$$

* Corresponding author: e-mail: david.mcdonnell@dit.ie, Phone: +00353863667487 
Examining orders of magnitude in equation (1) gives

$$
u \sim \beta \delta_{c}, \quad v \sim u \frac{\delta_{c}}{x}, \frac{\partial}{\partial x} \sim \frac{1}{x}, \frac{\partial}{\partial y} \sim \frac{1}{\delta_{c}}
$$

Now, the convection terms on the left hand side of equation (1) must be of the same order of magnitude as the diffusion term, $D \frac{\partial^{2} c}{\partial y^{2}}$. This gives

$$
\frac{\beta \delta_{c}}{x} \sim \frac{D}{\delta_{c}^{2}}
$$

which leads to

$$
\frac{\beta \delta_{c}^{3}}{x} \sim D
$$

The momentum boundary layer equation is

$$
u \frac{\partial u}{\partial x}+v \frac{\partial u}{\partial y}=\nu \frac{\partial^{2} u}{\partial y^{2}}-\frac{g c}{\rho}
$$

where $g$ is acceleration due to gravity, $\nu$ is the kinematic viscosity of the dissolution medium and $\rho$ is the density of the pure solvent. Performing a similar order of magnitude analysis as before gives

$$
u \frac{\partial u}{\partial x} \sim v \frac{\partial u}{\partial y} \sim \frac{\beta^{2} \delta_{c}^{2}}{x}
$$

and

$$
\nu \frac{\partial^{2} u}{\partial y^{2}} \sim \frac{\nu \beta}{\delta_{c}}
$$

This leads to

$$
\frac{u \frac{\partial u}{\partial x}}{\nu \frac{\partial^{2} u}{\partial y^{2}}} \sim \frac{\beta \delta^{3}}{\nu x}
$$

Now, from equation (4) we have $\frac{\beta \delta_{c}^{3}}{x} \sim D$, which gives

$$
\frac{u \frac{\partial u}{\partial x}}{\nu \frac{\partial^{2} u}{\partial y^{2}}} \sim \frac{D}{\nu} \sim \frac{1}{S_{c}}
$$

where $S_{c}$ is the Schmidt number. Now for liquids, $S_{c}>>1$, and therefore the inertia terms in equation (5) may be neglected with an error of order $\frac{1}{S_{c}}$ inside the concentration layer where the momentum boundary layer equation (5) reduces to

$$
\nu \frac{\partial^{2} u}{\partial y^{2}}=\frac{g c}{\rho}
$$

Integrating equation normally across the concentration boundary layer gives

$$
\nu\left[\frac{\partial u}{\partial y}\right]_{0}^{\delta_{c}}=\frac{g}{\rho} \int_{0}^{\delta_{c}} c d y
$$

which in turn leads to

$$
\left[\frac{\partial u}{\partial y}\right]_{\text {Blas }}-\beta=\frac{g}{\nu \rho} \int_{0}^{\delta_{c}} c d y
$$

where $\left[\frac{\partial u}{\partial y}\right]_{\text {Blas }}$ is the velocity gradient of the Blasius flow at $y=0$, taken from Schlichting[4]. Now, taking $C=\frac{c}{C_{s}}$ and rearranging (10) gives

$$
\beta=\frac{1}{3} U_{\infty} \sqrt{\frac{U_{\infty}}{\nu x}}-\frac{g C_{s}}{\nu \rho} \int_{0}^{\infty} C d y
$$


where $C_{s}$ is the concentration saturation. Finally, dividing across by $\left[\frac{\partial u}{\partial y}\right]_{\text {Blas }}$ and letting $T=\beta /\left[\frac{\partial u}{\partial y}\right]_{B l a s}$ gives

$$
T=1-\left[\frac{3 g C_{s}}{\mu} \sqrt{\frac{\nu x}{U_{\infty}^{3}}}\right] \int_{0}^{\infty} C d y
$$

It is clear that $\beta$ decreases with increasing $x$ and so we may introduce the method of Lévêque[1], which gives the forced heat transfer from a horizontal plate at large Prandtl numbers as a function of variable wall shear stress. When the appropriate changes have been made to the Lévêque solution, the following expression for the concentration of dissolved particles for flow over a flat plate may be deduced:

$$
C=h(\eta)=\frac{3}{\Gamma\left(\frac{1}{3}\right)} \int_{\eta}^{\infty} \exp \left(-\eta^{3}\right) d \eta
$$

where $\eta$ is given by

$$
\eta=\frac{y \sqrt{\beta}}{\left[9 D \int_{0}^{x} \sqrt{\beta} d x\right]^{\frac{1}{3}}}
$$

Introducing (13) and (14) into (12) gives

$$
T=1-\frac{3}{\Gamma\left(\frac{1}{3}\right)}\left[\frac{3 g C_{s}}{\mu} \sqrt{\frac{\nu x}{U_{\infty}^{3}}}\right]\left[\frac{\left[9 D \int_{0}^{x} \sqrt{\beta} d x\right]^{\frac{1}{3}}}{\sqrt{\beta}}\right] \int_{0}^{\infty} \eta \exp \left(-\eta^{3}\right) d \eta
$$

Noting that $\int_{0}^{\infty} \eta \exp \left(-\eta^{3}\right) d \eta=\frac{1}{3} \Gamma\left(\frac{2}{3}\right)$ and introducing a non-dimensional unit of length,$X=\frac{U_{\infty} x}{\nu}$, the above expression can be simplified to give

$$
T=1-\gamma\left[\frac{\left[\int_{0}^{X} T^{\frac{1}{2}} X^{-\frac{1}{4}} d X\right]^{\frac{1}{3}} X^{\frac{3}{4}}}{T^{\frac{1}{2}}}\right]
$$

where $\gamma=\frac{\Gamma\left(\frac{2}{3}\right)}{\Gamma\left(\frac{1}{3}\right)}\left[\frac{9 g C_{s} \sqrt[3]{\nu^{2} D}}{\rho U_{\infty}^{3}}\right]$. Finally to eliminate $\gamma$ we introduce $\tilde{X}=\gamma X$ and rearrange (16) to obtain

$$
(1-T)^{3} T^{\frac{3}{2}}=\tilde{X}^{\frac{9}{4}} \int_{0}^{\tilde{X}} T^{\frac{1}{2}} \tilde{X}^{-\frac{1}{4}} d \tilde{X}
$$

The integral equation (17) is solved by expanding $T$ in the power series $T=1+\sum_{n=1}^{\infty} a_{n} \tilde{X}^{n}$ and equating coefficients of $\tilde{X}$; this is extended to terms of order $\tilde{X}^{5}$. This leads to the following relationship between the non-dimensional shear stress, $T$, and $\tilde{X}$ :

$$
T \doteq 1-1.10 \tilde{X}-0.52 \tilde{X}^{2}-0.69 \tilde{X}^{3}-1.17 \tilde{X}^{4}-2.24 \tilde{X}^{5}
$$

neglecting terms of order $\tilde{X}^{6}$. Figure 1 shows successive polynomial approximations of equation (18). These results show convergence to the curve on the far left and indicate that separation occurs when $\tilde{X}$ is about one half, where

$$
\tilde{X}=9\left[\frac{\Gamma\left(\frac{2}{3}\right)}{\Gamma\left(\frac{1}{3}\right)}\right]\left[\frac{g x}{U_{\infty}^{2}}\right]\left[\frac{C_{s}}{\rho}\right]\left[\frac{1}{S_{c}}\right]^{\frac{1}{3}}
$$

Equation (19) may be rearranged to give

$$
F_{r}=\left[\frac{9 \Gamma\left(\frac{2}{3}\right) C_{s}}{\Gamma\left(\frac{1}{3}\right) \rho \tilde{X}}\right]^{\frac{1}{2}}\left[\frac{1}{S_{c}}\right]^{\frac{1}{6}}
$$

where $F_{r}$ is the non-dimensional Froude number, defined by $F_{r}=\frac{U_{\infty}}{\sqrt{g x}}$. Noting that $\Gamma\left(\frac{2}{3}\right) \approx 1.3541, \Gamma\left(\frac{1}{3}\right) \approx 2.6789$ and that separation occurs at $\tilde{X}=0.5$, the criterion for separation is given in terms of the Froude number as

$$
F_{r} \doteq 3\left[\frac{C_{s}}{\rho}\right]^{\frac{1}{2}}\left[\frac{1}{S_{c}}\right]^{\frac{1}{6}}
$$




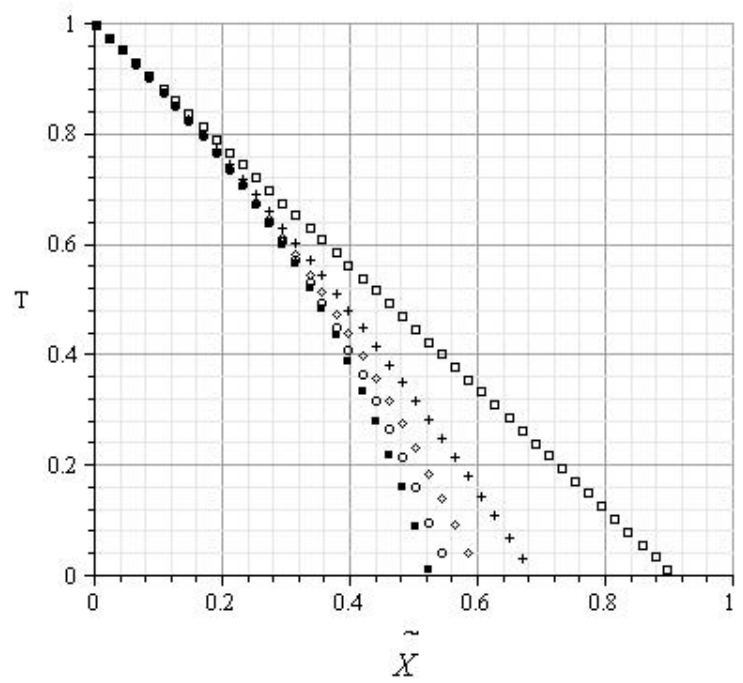

Fig. 1 Graph of Polynomial Approximations to Equation(18)

\section{Calculation of Flux from Surface due to Upward Flow below Separation Level}

In this section the total flux due to the upward velocity is calculated. From equation (13) we have

$$
C=\frac{3}{\Gamma\left(\frac{1}{3}\right)} \int_{\eta}^{\infty} \exp \left(-\eta^{3}\right) d \eta
$$

Differentiating with respect to $y$ gives

$$
\frac{\partial C}{\partial y}=\frac{3}{\Gamma\left(\frac{1}{3}\right)} \frac{\partial \eta}{\partial y} \frac{d}{d \eta}\left[\int_{\eta}^{\infty} \exp \left(-\eta^{3}\right) d \eta\right]
$$

Now the total flux from the surface is given as

$$
\begin{aligned}
\text { Flux / Unit Area } & =D\left[\frac{\partial c}{\partial y}\right]_{y=0} \\
& =\alpha_{1}\left[\frac{\sqrt{\beta}}{\left[\int_{0}^{x} \sqrt{\beta} d x\right]^{\frac{1}{3}}}\right]
\end{aligned}
$$

where $\alpha_{1}=D^{2 / 3} C_{s} \sqrt[3]{3} \frac{1}{\Gamma\left(\frac{1}{3}\right)}$. The total flux per unit width is given by

$$
\text { Flux / Unit Width }=\alpha_{1} \int_{0}^{x} \frac{\sqrt{\beta}}{\left[\int_{0}^{x} \sqrt{\beta} d x\right]^{\frac{1}{3}}} d x
$$

Equation (25) can be integrated by substituting $z=\int_{0}^{x} \sqrt{\beta} d x$ to give

$$
\text { Flux / Unit Width }=\frac{3}{2} \alpha_{1}\left[\int_{0}^{x} \sqrt{\beta} d x\right]^{\frac{2}{3}}
$$

Now, $\beta=T\left[\frac{\partial u}{\partial y}\right]_{\text {Blas }}$. This leads to

$$
\sqrt{\beta}=\sqrt{\frac{1}{3 \nu}} U_{\infty} \gamma^{\frac{1}{4}} \tilde{X}^{-\frac{1}{4}}\left[1-1.10 \tilde{X}-0.52 \tilde{X}^{2}-0.69 \tilde{X}^{3}-1.17 \tilde{X}^{4}-2.24 \tilde{X}^{5}\right]^{\frac{1}{2}}
$$


By applying the binomial expansion, equation (27) becomes

$$
\sqrt{\beta}=\sqrt{\frac{1}{3 \nu}} U_{\infty} \gamma^{\frac{1}{4}}\left[\tilde{X}^{-\frac{1}{4}}-0.55 \tilde{X}^{\frac{3}{4}}-0.26 \tilde{X}^{\frac{7}{4}}-0.345 \tilde{X}^{\frac{11}{4}}-0.585 \tilde{X}^{\frac{15}{4}}-1.12 \tilde{X}^{\frac{19}{4}}\right]
$$

Substituting (28) into (26) gives

$$
\text { Flux / Unit Width }=\alpha_{2}\left[\int_{0}^{x}\left[\tilde{X}^{-\frac{1}{4}}-0.55 \tilde{X}^{\frac{3}{4}}-0.26 \tilde{X}^{\frac{7}{4}}-0.345 \tilde{X}^{\frac{11}{4}}-\ldots\right] d x\right]^{\frac{2}{3}}
$$

where $\alpha_{2}=\frac{3}{2} \alpha_{1} \sqrt[3]{\frac{U_{\infty}^{2}}{3 \nu}} \gamma^{\frac{1}{6}}$. Noting that $x=\frac{\nu \tilde{X}}{\gamma U_{\infty}}$, equation (29) becomes

$$
\text { Flux / Unit Width }=\alpha_{2}\left[\frac{\nu}{\gamma U_{\infty}} \int_{0}^{\tilde{X}}\left[\tilde{X}^{-\frac{1}{4}}-0.55 \tilde{X}^{\frac{3}{4}}-0.26 \tilde{X}^{\frac{7}{4}}-0.345 \tilde{X}^{\frac{11}{4}}-\ldots\right] d \tilde{X}\right]^{\frac{2}{3}}
$$

Performing the integration in equation (30) and substituting in for $\alpha_{2}$ gives the total flux per unit width as

$$
\text { Flux / Unit Width }=0.2625\left[\frac{D C_{s} \rho U_{\infty}^{3} \tilde{X}}{g}\right]^{\frac{1}{2}}\left[1.33-0.314 \tilde{X}-0.095 \tilde{X}^{2}-0.092 \tilde{X}^{3}-\ldots\right]^{\frac{2}{3}}
$$

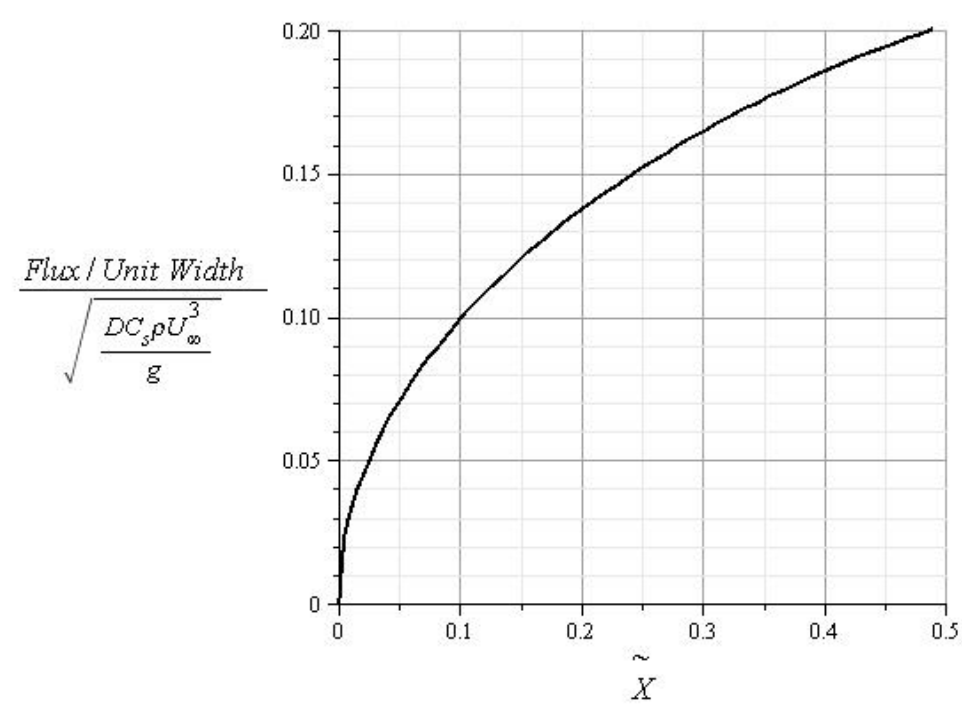

Fig. 2 Dependency of Flux per Unit Width on the variable $\tilde{X}$

\section{Effect of the Flow in the Concentration Layer on the Outer Layer}

From equation (14) it is easily shown that the ratio of the inner concentration layer thickness to that of the outer boundary layer is of order $S_{c}^{-\frac{1}{3}}$. Thus as $S_{c}$ tends to infinity, the outer limit of the velocity in the concentration layer is equal to the inner limit of the velocity in the outer layer, Van Dyke [5]. This velocity may be found by expressing equation (8) in terms of $\eta$, and solving the resulting equation using (22) for the concentration. It follows that the outer boundary layer is similar to that due to a stretching plate with an upward velocity $U_{\infty}$ just outside the boundary layer, the ratio of the plate velocity to $U_{\infty}$ being of order

$$
\left[\frac{g x}{U_{\infty}^{2}}\right]\left[\frac{C_{s}}{\rho}\right]\left[S_{c}\right]^{-\frac{2}{3}}
$$

and so for the values of the physical quantities discussed in this paper the Blasius flow is sensibly unchanged. 


\section{Discussion}

This work has its origins in the pharmaceutical industry; more specifically in the area of drug dissolution testing of solid dosage forms. Often in the testing of dissolution rates from solid dosage forms the soluble material is subject to a vertical flow. Typical values for a tablet composed of Benzoic acid placed in water are $C_{s}=4.564 \times 10^{-3} \mathrm{~g} / \mathrm{cm}^{3}, \mathrm{D}=1.236 \times$ $10^{-5} \mathrm{~cm}^{2} / \mathrm{s}$ and $\nu=0.7 \times 10^{-2} \mathrm{~cm}^{2} / \mathrm{s}$, as reported by D'Arcy[2]. Using these values Table (1) gives the distance along the surface at which boundary layer separation occurs for several outer stream velocities in the range $0 \leq U_{\infty} \leq 10 \mathrm{~cm} / \mathrm{s}$. The typical diameter of a cylindrical tablet is about $1 \mathrm{~cm}$ and Table (1) shows that, for an upward flow along a vertical flat plate of this height where mass transfer occurs, the upward flow will only begin to have a significant effect on the surface when the outer stream velocity is greater then $1 \mathrm{~cm} / \mathrm{s}$. That is to say, for velocities less then this value the boundary layer will separate, due to the weight of the dissolved particles, at relatively short distances from the leading edge. For sufficiently

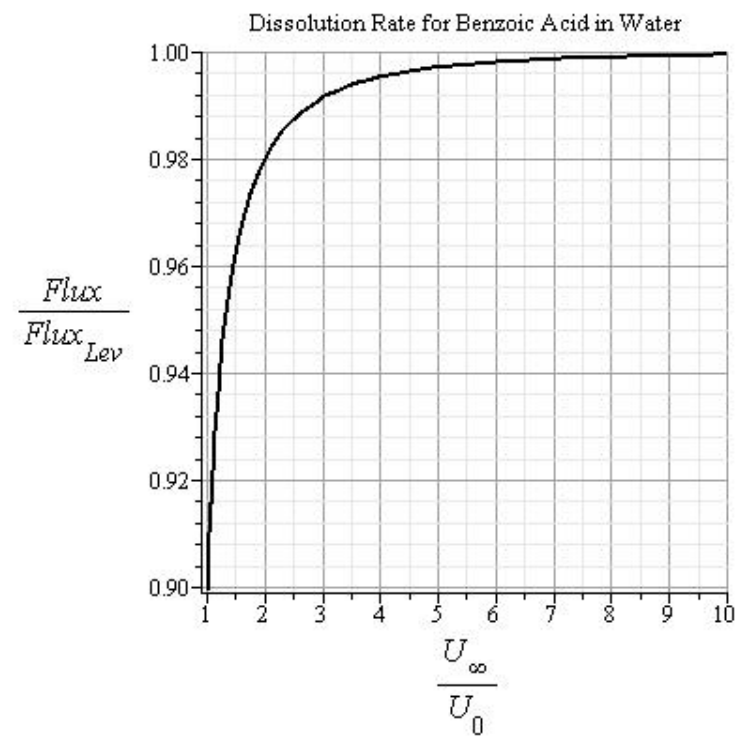

Fig. 3 Proportional Flux vs Non-Dimensional Velocity $\left(U_{\infty} \geq U_{0}\right)$

large upward velocities separation of the boundary layer will not occur. For the case of a Benzoic acid compact of diameter $1 \mathrm{~cm}$ dissolving in water, the criterion to prevent separation occurring across the height of the compact can be calculated from equation (21) to be $F_{r}>0.071$ In this instance, forced convection accounts for all of the mass transfer from the surface and equation (31) may be used to calculate the total flux from the surface. For a plate of given height, there exists a minimum upward velocity, namely $U_{0}$, required to prevent separation from occurring. This can be given in terms of the non-dimensional Froude number as $U_{0}=[g x]^{\frac{1}{2}} F_{r}$. Once this minimum velocity is exceeded, the mass transfer due to the upward velocity will approach the solution of Lévêque for horizontal flat plate flow, for which the flux from the surface is given by Schlichting[4] as

$$
\text { Flux / Unit Width }=0.677 \frac{U_{\infty}^{\frac{1}{2}} D^{\frac{2}{3}} C_{s} x^{\frac{1}{2}}}{\nu^{\frac{1}{6}}}
$$

Figure (3) shows the relationship between the non-dimensional velocity, $\frac{U_{\infty}}{U_{0}}$, and the flux per unit width from the surface of a soluble material as a proportion of the corresponding horizontal flow case.

Table 1 Point of Boundary Layer Separation for Various Velocities

\begin{tabular}{cccccccccc}
\hline$U_{\infty}(\mathrm{cm} / \mathrm{s})$ & 0.1 & 0.25 & 0.5 & 0.75 & 1 & 2.5 & 5 & 7.5 & 10 \\
\hline Separation Point $(\mathrm{cm})$ & 0.002 & 0.0127 & 0.0509 & 0.115 & 0.2037 & 1.273 & 5.093 & 11.459 & 20.372 \\
\hline
\end{tabular}




\section{References}

[1] M.A. Lévêque, Les lois de la transmission de chaleur par convection Ann Mines 13 , 201-239 (1928).

[2] D.M. D'Arcy, Hydrodynamic Simulation of Asymmetrically Positioned Tablets in the Paddle Apparatus: Impact on the Dissolution Rates and Variability Journal of Pharmacy and Pharmacology 57,1243 (2005).

[3] I.G. Currie, Fundamental Mechanics of Fluids 3rd Edition (CRC Press, Florida, 2003).

[4] H. Schlichting, Boundary Layer Theory 7th Edition (McGraw-Hill, New York, 1979).

[5] M. Van Dyke, Perturbation Methods in Fluid Mechanics (The Parabolic Press, Stanford, California, 1975). 